\title{
Perfil da clientela atendida em um serviço público de urgência psiquiátrica no município de Belo Horizonte, Brasil, no período de 2002 a 2007
}

Clinical and demographical profile of patients admitted to a psychiatric emergency service of Belo Horizonte, Brazil, 2002-2007

Fernando Madalena Volpe, Eliane Mussel da Silva', Leonardo Silva Carmo ${ }^{1}$, Terezinha Neila dos Santos ${ }^{1}$

\section{RESUMO}

Objetivo: Descrever as características sociodemográficas, o número e os tipos de atendimentos realizados em um serviço público de atendimento de Urgência Psiquiátrica de Belo Horizonte, Brasil, no período de 2002 a 2007. Métodos: Estudo descritivo de séries temporais, com análises de tendência por meio de regressões lineares, em que a variável resposta foi o número anual de atendimentos (totais e por tipo) ou a proporção de atendimentos por diagnóstico e a variável preditora foi o ano. Resultados: 0 número total de atendimentos no período pesquisado não apresentou alteração significativa, embora tenha ocorrido uma redução do número de primeiras consultas no serviço, com aumento do número de retornos. Foi observada redução dos diagnósticos associados a álcool e drogas de abuso e predominância dos transtornos psicóticos. Conclusão: No período estudado, o Hospital Galba Velloso - FHEMIG manteve o volume total de atendimentos, mas observou-se redução da proporção de

\section{Palavras-chave}

Atendimento à crise, saúde mental, clientela.

\section{Keywords}

Psychiatric urgency, mental health, clients. pacientes oriundos da capital, bem como redução da procura por diagnósticos associados a álcool e drogas de abuso, enquanto a categoria diagnóstica mais prevalente foi a dos transtornos psicóticos. Houve redução dos primeiros atendimentos e das internações prolongadas.

\section{ABSTRACT}

Objective: To describe the social-demographic characteristics, the number and the types of emergency visits during the period of 2002-2007 in a psychiatric public service of Belo Horizonte, Brazil. Methods: A time-series descriptive study with trend analysis through linear regressions, where the dependent variable was the annual number of visits (total and by type), or the proportion of visits for diagnosis, and predictor variable was year. Results: The total number of visits presented no significant changes. Nevertheless, a reduction on the number of first visits and an increase on the number of return consultations were detected. There was a reduction on the diagnosis associated with alcohol and drug abuse and a predominance of psychotic disorders. Admissions of patients residing in the capital were also reduced. Conclusion: The results indicate that the total number of visits during the study period remained unchanged, while there was a reduction of patients from the capital and of the proportion of first consultations and of alcohol/drug related disorders. The most frequent diagnosis was psychotic disorders and schizophrenia.

1 Fundação Hospitalar do Estado de Minas Gerais (FHEMIG), Núcleo de Ensino e Pesquisa do Hospital Galba Velloso (HGV).

$$
\begin{gathered}
\text { Recebido em } \\
12 / 3 / 2010 \\
\text { Aprovado em } \\
1 / 9 / 2010
\end{gathered}
$$

Endereço para correspondência: Fernando Madalena Volpe Alameda Álvaro Celso, 100 30150-260 - Belo Horizonte, MG, Brasil Telefone: (31) 3239-9545 E-mail: fernando.volpe@fhemig.mg.gov.br 


\section{INTRODUÇÃO}

O início do processo de reforma psiquiátrica no Brasil ao final dos anos 1970 surge em decorrência, por um lado, da crise no modelo que fundamentou os paradigmas da psiquiatria clássica e tornou o hospital psiquiátrico a única alternativa de tratamento, facilitando a cronicidade e a exclusão dos doentes mentais, e, por outro, dos esforços dos movimentos sociais pelos direitos dos pacientes psiquiátricos?.

A partir da década de 1990, o Ministério da Saúde define uma nova política de saúde mental em consonância com as recomendações da Organização Pan-Americana de Saúde (OPAS), redirecionando gradativamente os recursos da assistência psiquiátrica para um modelo "substitutivo". Incentiva-se a criação dos Centros de Atenção Psicossocial (CAPS), os Serviços Residenciais Terapêuticos (SRT), os Centros de Convivência e Cultura, leitos de atenção integral tanto em hospitais gerais como nos CAPS III, o programa De Volta para a Casa e o Programa de Saúde da Família (PSF)3.

Em Minas Gerais, a aprovação da lei nacional no 10.216, em 2001, veio fortalecer as leis estaduais no 11.802, de 1995, e no 12.684 , de 1997, que preconizam a extinção progressiva dos hospitais psiquiátricos e sua substituição por uma rede de serviços abertos e comunitários.

De fato, essa política vem sendo implantada no estado. Em 1991, segundo dados da Secretaria de Estado de Saúde de Minas Gerais, existiam 36 hospitais psiquiátricos em Minas, perfazendo um total de 8.087 leitos; já em dezembro de 2008, contava-se com 2.889 leitos em 20 hospitais4. Por outro lado, o número de CAPS implementados no estado vem aumentando a partir da década de 1990, totalizando, em dezembro de 2009, 145 Centros de Atenção Psicossocial, cuja clientela-alvo são os portadores de sofrimento mental severo e persistente acolhidos, sobretudo, nas situações de crise; cerca de 300 equipes de Saúde Mental na Atenção Básica; 12 Centros de Convivência; 35 Serviços Residenciais Terapêuticos e 3.983 Equipes de Saúde de Família4.

Para gestão e planejamento da cidade, Belo Horizonte, MG, é dividida em nove áreas administrativas que coincidem com os Distritos Sanitários, a saber: Distritos Sanitários Leste, Oeste, Nordeste, Noroeste, Centro-Sul, Sudeste, Barreiro, Pampulha e Venda Nova ${ }^{5}$.

Os CERSAM (Centros de Referência em Saúde Mental) foram sendo implementados em cada Distrito Sanitário, com o propósito de serem serviços substitutivos ao Hospital, em Belo Horizonte, a partir de 1993, com o início das atividades do CERSAM Barreiro, seguido pelos CERSAM Noroeste, em 1995, Leste, em 1996, Pampulha, em 1997, Nordeste, Venda Nova e Oeste, em 2002, totalizando sete CERSAM em funcionamento ${ }^{6}$.

Em 2002, o Projeto de Saúde Mental (PSM-BH) do município foi inserido no Programa "BH Vida: Saúde Integral", tendo como diretrizes e política a construção de uma rede integra- da de serviços, programas e ações inseridos na comunidade, capazes de desenvolver uma estratégia que respondesse às necessidades de saúde mental dos diversos territórios, a partir de uma perspectiva e prática de desinstitucionalização. Uma das premissas do projeto é que os Centros de Saúde se tornem a porta de entrada preferencial na rede para os portadores de transtornos mentais, funcionando com fluxo de referência e contrarreferência a outros serviços. É importante ressaltar que os CERSAM participam dessa rede de assistência e também servem de porta de entrada para o sistema.

Não obstante, mesmo diante da ampla e complexa rede de atenção à saúde mental, Belo Horizonte ainda conta com dois hospitais psiquiátricos públicos, administrados pela Fundação Hospitalar do Estado de Minas Gerais, para o atendimento em situação de crise de pacientes psiquiátricos acima de 18 anos de idade: o Instituto Raul Soares (IRS), atualmente com 111 leitos, e o Hospital Galba Velloso (HGV), com 145 leitos operacionais ${ }^{8}$.

Em sintonia com os ideais da Reforma Psiquiátrica Brasileira, em agosto de 2001 foi criado no Hospital Galba Velloso o Centro de Acolhimento da Crise (CAC). Sua função é o acolhimento e o tratamento de sujeitos em crise buscando a estabilização em um menor tempo possível e a inserção do paciente para tratamento externo na rede de atenção à saúde mental $\left.\right|^{9}$. A partir de 2002, pacientes atendidos no CAC que necessitavam de internação não podiam mais ser encaminhados a outros hospitais conveniados ao Sistema Único de Saúde (SUS).

No que diz respeito ao atendimento das urgências/emergências psiquiátricas, o modelo adotado em 2001 pela Secretaria Municipal de Saúde foi para oferecer dois CERSAM 24 horas, com 6 leitos-noite em cada serviço, dando respostas à crise seja por demandas espontâneas ou trazidas pelo SAMU (Serviço de Atendimento Móvel de Urgência) ou pela Polícia Militar. Em 2006, buscando uma melhor integração e construção de uma rede de atendimento às situações de crise, foi implantada a Hospitalidade Noturna em todos os sete CERSAM e criado o Serviço de Urgência Psiquiátrica (SUP), localizado na região central da cidade, com seis leitos e funcionando das 19 às 7 horas.

No projeto de atenção à saúde mental, como visto, a resposta à crise deve ser objeto de pronto acolhimento e acesso prioritário aos "serviços substitutivos".

Os avanços da trajetória da reforma psiquiátrica brasileira podem ser observados nas análises de autores ${ }^{10,11}$ que convergem ao apontar que o processo em curso no país consiste na redução do número de leitos e hospitais psiquiátricos com a criação de serviços extra-hospitalares.

Se, por um lado, os dados institucionais mostram redução do número de leitos em hospitais psiquiátricos e aumento progressivo do número de CAPS, por outro lado não tem sido considerada outra variante importante na avaliação desse novo modelo de assistência: o número e o perfil 
dos atendimentos nas urgências dos hospitais psiquiátricos, como indicadores da resolutividade dos "serviços substitutivos". Uma abordagem simplista da desospitalização carrega um potencial viés ao desconsiderar a hipótese de que, mesmo com o desenvolvimento e o amadurecimento dos serviços extra-hospitalares de atendimento à saúde mental, pacientes com um perfil específico com transtornos mentais especialmente graves venham a necessitar da assistência hospitalar, ainda que de curta duração. Se verdadeira, a hipótese acima desafiaria o conceito de "serviços substitutivos" atribuído aos serviços extra-hospitalares, indicando uma estrutura complementar de atenção à saúde mental que incluiria todos os níveis de assistência. Para compreender mais detalhadamente qual o papel do hospital psiquiátrico diante da reforma à assistência à saúde mental, uma primeira abordagem é a descrição do perfil e do volume dos atendimentos efetuados por ele ao longo do processo de estruturação da rede extra-hospitalar.

Seguindo essa lógica, este estudo visa descrever a evolução temporal dos atendimentos realizados no CAC-HGV de 2002 a 2007, explorando mudanças no volume de atendimentos e no perfil dos pacientes atendidos.

\section{MATERIAL E MÉTODOS}

Este é um estudo descritivo, de séries temporais, baseado nos dados disponibilizados pelo Serviço de Informação do HGV, onde foram registrados todos os atendimentos desde o ano de 2002. Como rotina administrativa do Hospital, as informações do paciente são cadastradas no Serviço de Informação/HGV quando da chegada do paciente à instituição e são concluídos todos os campos quando da alta dele pelo funcionário do Serviço de Prontuários do Paciente (SPP) a partir dos dados do prontuário do paciente, como da folha de alta e encaminhamento médico.

Foram coletados os dados relativos às características sociodemográficas da população atendida no Centro de Acolhimento da Crise/HGV (sexo, idade, procedência), bem como o número de atendimentos realizados, número de pacientes atendidos, número de retornos, hipótese diagnóstica e tipo de atendimento realizado (consulta, observação, enfermaria de crise ou enfermaria de média permanência). Ainda quanto ao tipo de atendimento, foi considerado como retorno todo atendimento de paciente ocorrido no mesmo ano da visita de referência, independentemente do intervalo entre as visitas. As hipóteses diagnósticas levantadas foram codificadas, para fins de registro estatístico, de acordo com a $10^{a}$ edição da Classificação Internacional de Doenças (CID-10). No presente artigo, foi utilizada apenas a primeira impressão diagnóstica levantada, por considerar que esse seria o diagnóstico principal associado com a visita ao CAC-HGV.
Para a análise descritiva dos atendimentos realizados, foram computadas proporções para as variáveis categóricas e médias e respectivos desvios-padrão para as contínuas. Para a comparação das idades médias dos pacientes atendidos em 2002 e 2007, utilizou-se o teste $t$ de Student para observações independentes. Para a comparação das proporções por sexo e por procedência dos atendimentos efetuados em 2002 e 2007, foi utilizado o teste de qui-quadrado para heterogeneidade. As análises de tendência foram realizadas mediante regressões lineares simples (e quadráticas, quando significativas), em que a variável resposta era o número anual de atendimentos (totais e por tipo) ou a proporção de atendimentos por diagnóstico e a variável preditora era o ano. Para evitar a autocorrelação entre os termos da equação de regressão, foi utilizado o artifício de centralizar a variável ano, transformando-a em ano-calendário menos 2004,5 (ano 2004,5), pois esse era o ponto médio da série histórica. Em todos os casos, o alfa foi estabelecido em 5\%, bicaudado.

O presente estudo recebeu a aprovação do Comitê de Ética em Pesquisa da FHEMIG.

\section{RESULTADOS}

\section{Número total de atendimentos por ano}

Em 2002, o número total de atendimentos foi de 10.561, chegando a 9.731 em 2007. Essa aparente redução, no entanto, não foi significativa $(p=0,416)$.

\section{Tipo de atendimento}

O número de atendimentos realizados no Centro de Acolhimento da Crise (CAC) do HGV, em todo o período pesquisado, foi de 60.139, correspondendo a uma média de 716 atendimentos mensais. Desse total, 67,3\% (40.451) foram atendidos pela primeira vez na instituição e 32,7\% (19.688) dos atendimentos dizem respeito aos retornos. Observou-se redução linear do número de primeiros atendimentos entre 2002 e 2007, estatisticamente significativa, de 245 atendimentos por ano (média anual $=6.742 ; R 2=74,3 \% ; p=0,017$ ). Simultaneamente, observou-se aumento significativo de 163 retornos ao ano no período (média anual $=3.281$; R2 $=68 \%$; $p=0,027$ ) (Figura 1).

Em relação ao tipo de atendimento realizado segundo o tempo de permanência hospitalar (Figura 2), observou-se redução linear significativa no número de atendimentos externos (permanência na instituição inferior a 4 horas), de 160 atendimentos ao ano (média anual $=5.821 ; R 2=60,6 \% ; p=$ 0,042); aumento do número de observações clínicas (permanência de 4 a 24 horas), de 122 ao ano (média anual = 1.319; $\mathrm{R} 2=89,7 \% ; \mathrm{p}=0,03$ ); aumento das internações no CAC (acima de 24 horas), de 57,2 ao ano (média anual = 1.855; R2 = $81,4 \% ; p=0,009)$ e distribuição quadrática $[Y=913-87,1 \times$ 
$\left(\right.$ ano-2004,5) + 45,4 x (ano-2004,5) $\left.{ }^{2}\right]$ das internações nas enfermarias do HGV (R2 =96,4\%; $p=0,003)$. O número de internações nas enfermarias do HGV não se correlacionou com a redução de 147 para 100 leitos do HGV observada entre 2002 e 2007 ( $r=0,68 ; p=0,138)$. Nesse mesmo período, o número de leitos psiquiátricos do SUS em Belo Horizonte também se reduziu de 1.280 para 1.034, sem correlação com o número de internações no HGV $(r=0,85 ; p=0,143)$.

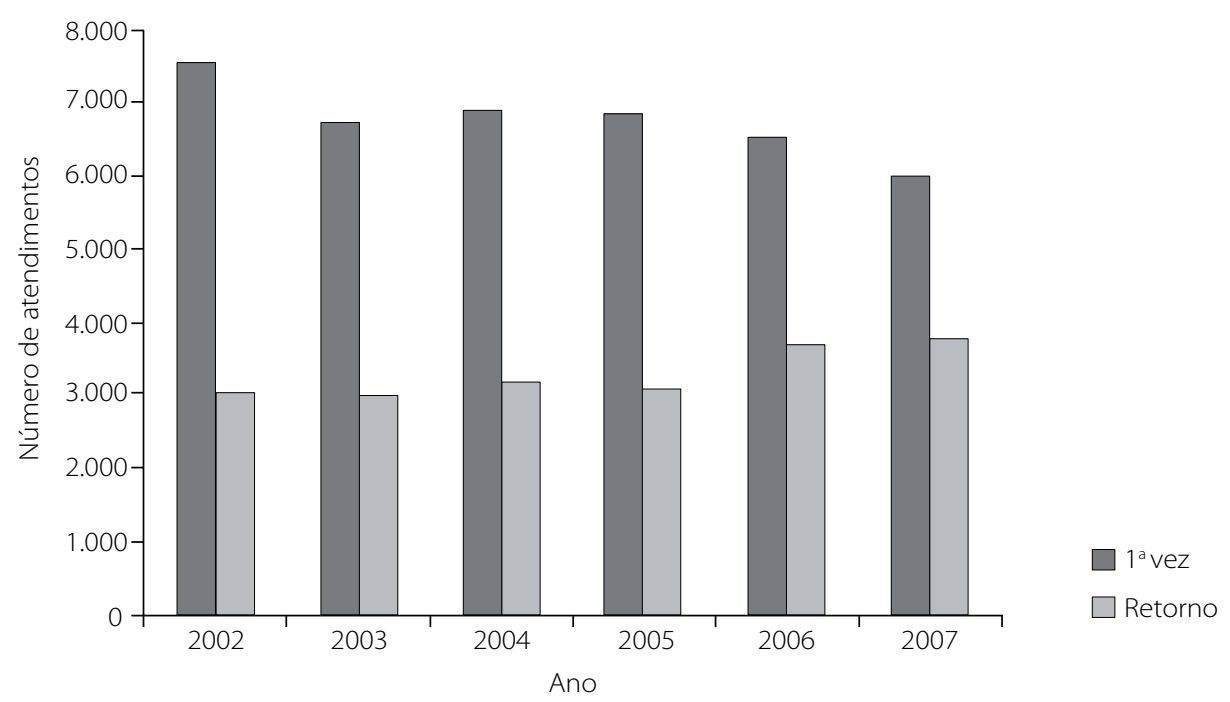

Figura 1. Número de primeiras consultas e retornos, HGV, 2002-2007.

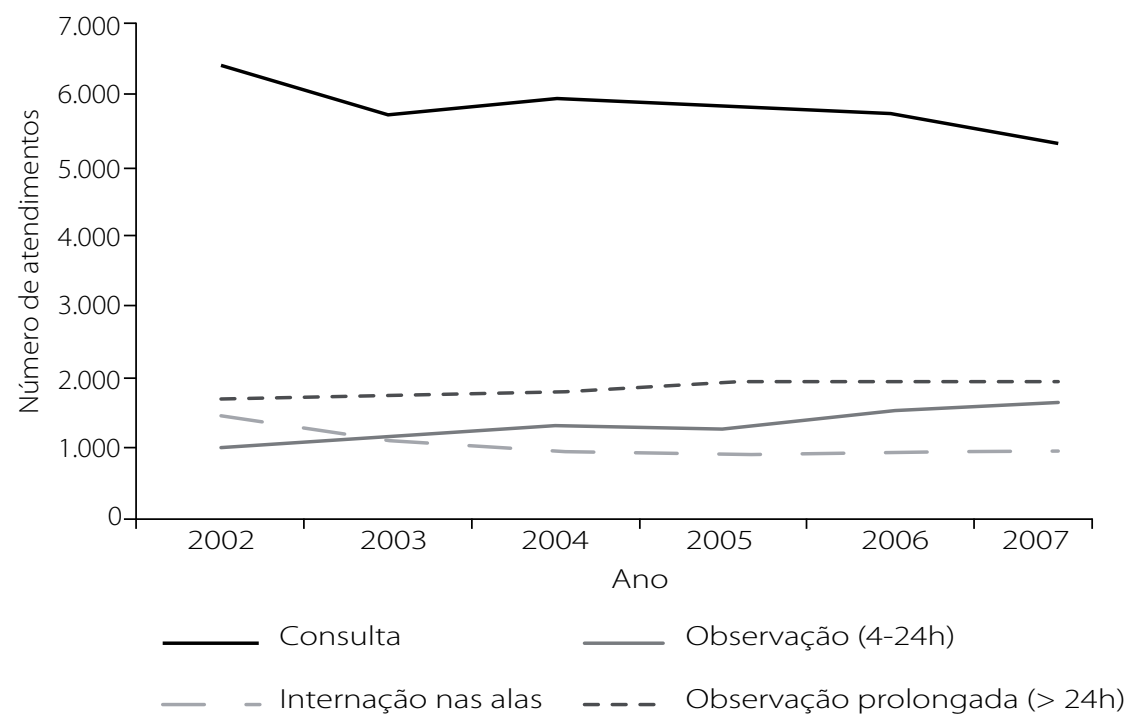

Figura 2. Número de atendimentos segundo o tempo de permanência na instituição, HGV, 2002-2007.

\section{Procedência}

Dos 40.451 pacientes atendidos no CAC-HGV durante todo o período pesquisado, 45,4\% foram procedentes de Belo Horizonte, 39,4\%, da região metropolitana, 15,0\%, oriundos de outras cidades do estado de Minas Gerais e 0,2\%, de outros estados (Figura 3). Entre 2002 e 2007, foi observada redução significativa da proporção dos atendimentos a pacientes procedentes de Belo Horizonte (48,3\% para 38,0\%), enquanto a proporção de pacientes atendidos procedentes das ou- tras localidades da região metropolitana subiu de 35,0\% para $45,0 \%\left(X^{2}=139,7 ; D F=3 ; p<0,001\right)$.

\section{Idade e sexo}

No inteiro período de 2002 a 2007, 58,6\% das pessoas atendidas no serviço foram do sexo masculino e a idade média foi de 38,6 ( $\pm 12,7)$ anos. A faixa etária mais prevalente foi aquela entre 31 e 40 anos (28,4\%). Não houve mudanças significativas no perfil etário e distribuição por sexos, entre 2002 e 2007. 


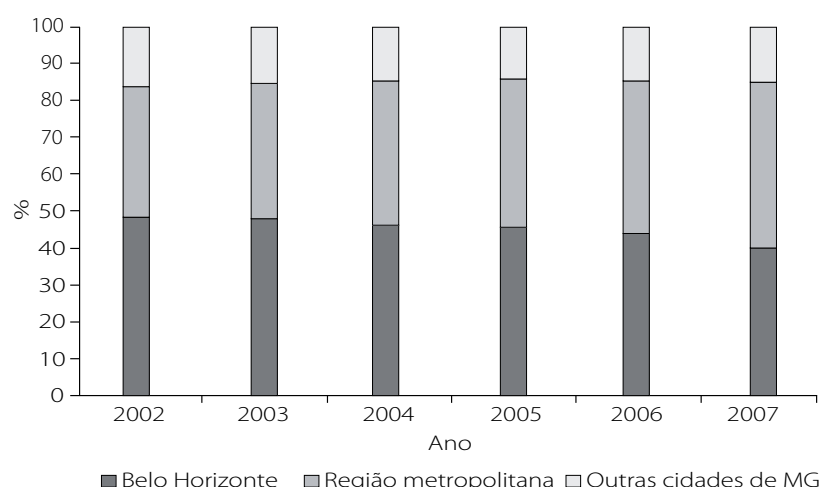

Figura 3. Distribuição da clientela atendida por procedência, HGV, 2002-2007.

\section{Diagnósticos}

Dos 60.139 atendimentos realizados em todo o período pesquisado, 31,3\% tinham o diagnóstico de esquizofrenia, transtornos esquizotípicos e delirantes (F20-F29); 21,5\%, transtornos mentais e de comportamento decorrentes do uso de álcool/substâncias psicoativas (F10-F19); 16,5\%, transtornos do humor (afetivos) (F30-F39); 12,6\%, transtornos neuróticos, relacionados ao estresse e somatoformes (F40-F49), e 4,7\% não tinham hipótese diagnóstica.

Entre 2002 e 2007, a proporção de pacientes atendidos com transtornos decorrentes do uso de álcool/substâncias psicoativas caiu $0,6 \%$ a cada ano $\left(R^{2}=68,6 \% ; p=0,026\right)$ (Figura 4). As frequências de outras categorias diagnósticas psiquiátricas não variaram significativamente, a não ser o grupo sem hipótese diagnóstica, que cresceu significativamente em uma taxa de 0,9\% ao ano ( $R 2=86,3 \% ; p=0,005)$.

\section{DISCUSSÃO}

De acordo com nosso conhecimento, esta é a primeira descrição quantitativa e evolutiva das características do paciente que procura a urgência psiquiátrica, no contexto da reforma à assistência em saúde mental em curso no país na última década. A heterogeneidade entre as reformas na assistência conduzidas nos diversos países dificulta a comparação objetiva de estratégias ${ }^{12-17}$. Nesse contexto, a necessidade de produzir dados relevantes para a avaliação das mudanças nos serviços de saúde mental já foi apontada por outros autores ${ }^{18,19}$ e constitui a essência do presente artigo.

Os resultados deste estudo mostram que no HGV o número global de atendimentos se manteve entre 2002 e 2007, apesar de no mesmo período terem ocorrido importantes mudanças e ampliação na rede de atenção à saúde mental no município de Belo Horizonte como implantação do serviço noturno em todos os Centros de Referência em Saúde Mental (CERSAM), a criação do Serviço de Urgência Psiquiátrica Municipal (SUP) e do Serviço de Atendimento Médico de Urgência (SAMU) e o amadurecimento da rede de equipes de saúde da família. Diante disso, duas hipóteses merecem posterior avaliação em estudos específicos: 1) que os casos graves continuam necessitando da assistência hospitalar, não obtendo resolutividade no atendimento extra-hospitalar; e/ou 2) que as mudanças ocorridas no sistema de atenção à saúde mental na região metropolitana de Belo Horizonte ainda não foram assimiladas pela população, que continua considerando o hospital como recurso para a urgência psiquiátrica. Para avançar na exploração desta, um estudo qualitativo está sendo conduzido para compreender as motivações de usuários e familiares para buscar a assistência no CAC-HGV.

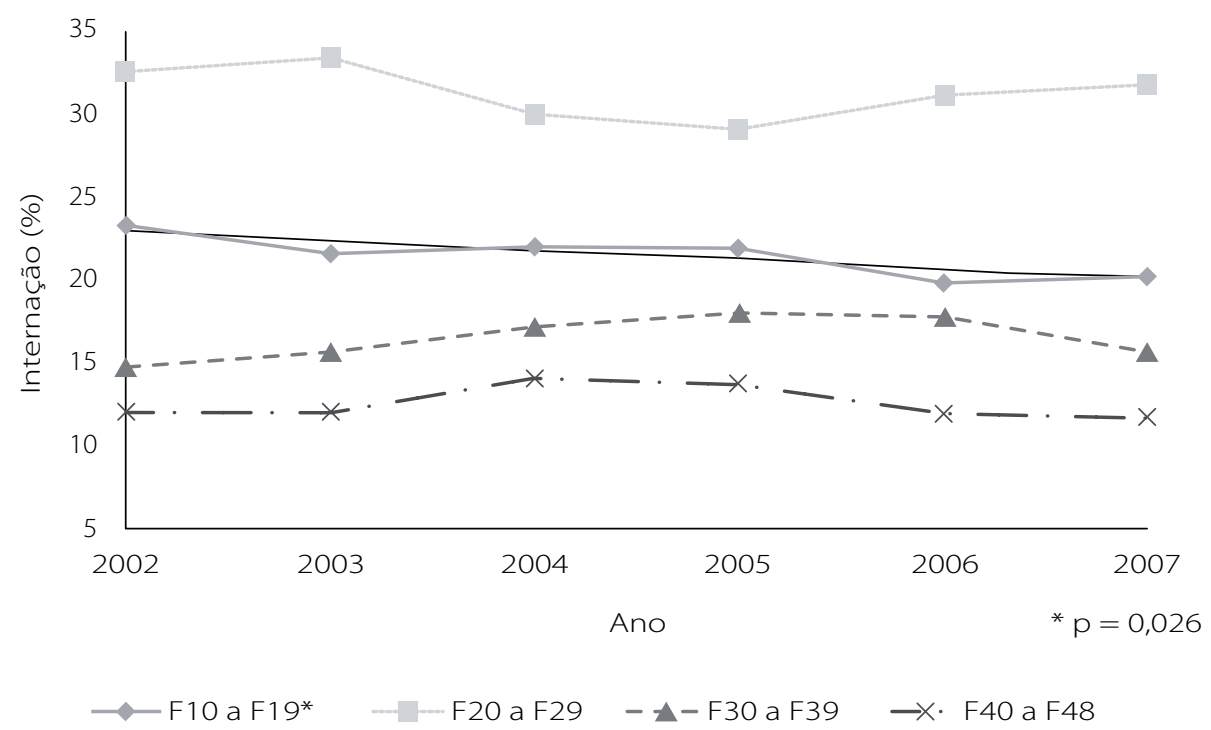

Figura 4. Distribuição dos atendimentos por diagnóstico, HGV, 2002-2007. 
A redução do número de pacientes que procuraram o serviço pela primeira vez, aliada à redução de pessoas que buscam o serviço procedentes da cidade de Belo Horizonte, aponta para a hipótese de que a rede de atenção à saúde mental do município esteja absorvendo a população que necessita de atendimento durante a situação de crise. Por outro lado, houve aumento do número de pacientes que retornam ao hospital no mesmo ano, um indicativo de recaída ou recorrência. Tomados em conjunto, esses resultados levantam a possibilidade da existência de falhas no referenciamento dos pacientes do hospital para a rede. Essa hipótese, no entanto, deverá ser verificada em novos estudos que avaliem diretamente o impacto do acesso aos serviços extra-hospitalares sobre a intensidade da procura pelo hospital.

O aumento relativo observado na proporção de pacientes provenientes dos outros municípios da região metropolitana aponta para uma possível desproporção entre o aumento da população e o insuficiente dimensionamento da rede extra-hospitalar de saúde mental nas cidades vizinhas a Belo Horizonte ${ }^{20}$, ou seja, a rede extra-hospitalar nos municípios vizinhos não foi capaz de conter ou evitar a crise fora do hospital com a mesma eficiência que a da capital.

No período pesquisado, houve uma redução significativa das internações em enfermarias de média permanência, com aumento do número de observações clínicas (permanência no serviço de 4 a 24 horas) e internações no CAC (acima de 24 horas), com redução dos atendimentos externos. A redução das internações nas enfermarias de média permanência reforça a hipótese de que a eficiência dos serviços de urgências psiquiátricas contribui para diminuir as admissões hospitalares desnecessárias. Ainda, na medida em que os serviços de urgências estão integrados com os serviços extra-hospitalares, promovem aumento da qualidade do sistema de saúde como um todo, levando à redução das "consultas" (atendimento externo) nos serviços de urgência, como visto no HGV.

A categoria diagnóstica mais frequente nesta amostra de atendimentos de urgência psiquiátrica foi "esquizofrenia, transtornos esquizotípicos e delirantes" (F20-F29). Essa predominância é observável considerando todas as internações hospitalares por transtornos mentais e do comportamento, em Belo Horizonte ou em todo o estado de Minas Gerais, mantendo-se a principal causa de internação de 2002 a 2007 (Datasus) $^{20}$. Transtornos ligados ao uso de álcool e outras drogas foram a segunda causa mais frequente de atendimentos neste serviço, com tendência decrescente. No mesmo período, considerando todo o estado, a proporção das internações por transtornos ligados ao uso de álcool e outras drogas (F10-F19) se reduziu até 2006 (de 31\% a 25\%), voltando a se elevar em 2007 (30\%). Comparando esses achados com os resultados do resto do país (excluindo MG) para o período de 2002-2007 (Datasus) 20, observam-se uma tendência à re- dução proporcional nas internações por F20-F29 (de 45\% a 39\%) e uma elevação nos casos de F10-F19 (de 31\% a 34\%). Observa-se, por meio dos dados oficiais, que as tendências são variáveis também segundo os estados; por exemplo, no Rio de Janeiro houve também uma importante redução nas internações por F10-F19 entre 2002-2007 (de 26\% a 20\%), sem grandes mudanças na proporção de internações por F20-F29 ( 54\%). Em São Paulo, no mesmo período, não se observou alteração importante das proporções de internações por F20-F29 ( 35\%) nem de F10-F19 ( 37\%). O Datasus é um sistema on-line de informações em saúde mantido e disponibilizado pelo Ministério da Saúde do Brasil, condensando dados fornecidos pelas secretarias estaduais e municipais de saúde, bem como outros indicadores de importância para o setor da saúde. Essas informações podem servir para subsidiar análises objetivas da situação sanitária, tomadas de decisão baseadas em evidências e elaboração de programas de ações de saúde. Nesse sistema, o registro de morbidade hospitalar é baseado no diagnóstico primário que motivou a internação, apenas dos hospitais participantes do Sistema Único de Saúde. Por se tratar de dados secundários, há possibilidade de registros inadequados. Ainda não há estudos mensurando a acurácia dos registros de diagnóstico do Sistema de Informações Hospitalares (SIH) do Datasus referente à saúde mental. É improvável, no entanto, que isso gere erros sistemáticos nas estimativas das prevalências dos diagnósticos primários. Por outro lado, os dados disponibilizados não incluem a comorbidade, por exemplo, de dependência ou abuso de álcool e drogas com outros transtornos mentais, impossibilitando a estimativa real da prevalência de F10-F19 entre a população atendida.

Essas diferenças de diagnósticos que motivaram a internação observadas entre os estados não podem ser atribuídas a prevalências distintas dos transtornos psicóticos ou das dependências/abusos de substâncias e álcool, pois os estudos epidemiológicos multicêntricos nacionais revelaram taxas de prevalência consistentes ${ }^{21,22}$. Pode-se especular que essa heterogeneidade seja resultante de fatores ligados com o acesso dos pacientes aos serviços hospitalares e extra-hospitalares psiquiátricos, bem como aos perfis de conduta e atendimento possivelmente variáveis entre os serviços de cada estado e que determinariam a decisão de internar ou não.

Merece destaque a proporção elevada de pacientes do sexo masculino entre os portadores de esquizofrenia ou transtornos psicóticos atendidos na urgência psiquiátrica do HGV. Já foi hipotetizado que as mulheres portadoras de psicoses teriam melhor adesão ao tratamento nos serviços extra-hospitalares ${ }^{23-25}$. É possível supor que nos homens a psicose curse com uma maior impulsividade e agressividade, o que justificaria a maior demanda desses pacientes por tratamento hospitalar. 


\section{CONCLUSÃO}

No período estudado (2002-2007), o Hospital Galba Velloso - FHEMIG manteve o volume total de atendimentos, mas observou-se redução da proporção de pacientes oriundos da capital, bem como redução da procura por diagnósticos associados a álcool e drogas de abuso, enquanto a categoria diagnóstica mais prevalente foi a dos transtornos psicóticos. Houve redução dos primeiros atendimentos e das internações prolongadas.

\section{AGRADECIMENTOS}

À Fundação de Amparo à Pesquisa do Estado de Minas Gerais (Fapemig), pelo apoio financeiro, e a Felipe Antônio Fernandes Gontijo e Carina Ribeiro de Aquino, bolsistas de iniciação à pesquisa, pela coleta de dados.

\section{REFERÊNCIAS}

1. Gonçalves AM, Sena RR. A reforma psiquiátrica no Brasil: contextualização e reflexos sobre 0 cuidado com o doente mental na família. Rev Lat Am Enfermagem. 2001;9(2):48-55.

2. Brasil. Ministério da Saúde. Secretaria de Atenção à Saúde. DAPE. Coordenação Geral de Saúde Mental. Reforma psiquiátrica e política de saúde mental no Brasil. Documento apresentado à Conferência Regional de Reforma dos Serviços de Saúde Mental: 15 anos depois de Caracas. OPAS. Brasília, novembro de 2005.

3. Delgado PG, Gomes MP, Coutinho ES. Novos rumos nas políticas públicas da saúde mental no Brasil. Cad Saude Publica. 2001;17(3):452-3.

4. Minas Gerais. Secretaria de Estado de Saúde. Atenção em Saúde Mental. Saúde em Casa. 1a edição. Belo Horizonte; 2006, p. 36.

5. Belo Horizonte. Secretaria Municipal de Saúde de Belo Horizonte. Avanços e desafios na organização da atenção de saúde em Belo Horizonte. Org. Maria Aparecida Turci. Belo Horizonte: HMP Comunicação; 2008, p. 44-83

6. Oliveira GL. Saúde mental e a continuidade do cuidado em Centros de Saúde de Belo Horizonte [dissertação]. Faculdade de Medicina da Universidade Federal de Minas Gerais; 2006.

7. Belo Horizonte. Propostas da Secretaria Municipal de Saúde. VIII Conferência Municipal de Saúde: etapa municipal da XII Conferência Nacional: Belo Horizonte; 2003.
8. Belo Horizonte. Fundação Hospitalar de Minas Gerais. Censo informação hospitalar. Capacidade hospitalar operacional. Disponível em: http://10.49.10.6/censo/armazem00a2.php. Acessado em: 22/10/2009.

9. Mascarenhas MEB. Relatório de atividades e proposta de trabalho da diretoria clínica a da chefia de divisão técnico-assistencial do Hospital Galba Velloso para o ano de 2000. Belo Horizonte; 1999.

10. Tenório F. A reforma psiquiátrica brasileira, da década de 1980 aos dias atuais: história e conceito. História, Ciências, Saúde - Manguinhos. Rio de Janeiro, v. 9, n. 1, 2002.

11. Alves DS. 0 "Ex" - tentando ver o futuro. Cadernos IPUB. Rio de Janeiro, n. 14, p. 21-30, 1999.

12. Short T, Thomas S, Luebbers S, Ogloff JR, Mullen P. Utilization of public mental health services in a random community sample. Aust N Z J Psychiatry. 2010;44(5):475-81.

13. Lora A. An overview of the mental health system in Italy. Ann Ist Super Sanita. 2009;45(1):5-16

14. Preti A, Rucci P, Santone G, Picardi A, Miglio R, Bracco R, et al. Patterns of admission to acute psychiatric in-patient facilities: a national survey in Italy. Psychol Med. 2009;39(3):48596. Epub 2008 Jun 26

15. Muijen M. Focus on mental health care reforms in Europe. Mental health services in Europe: an overview. Psychiatry Serv. 2008;59(5):479-82.

16. Becker T, Kilian R. Psychiatric services for people with severe mental illness across Western Europe: what can be generalized from current knowledge about differences in provision, costs and outcomes of mental health care? Acta Psychiatr Scand Suppl. 2006;(429):9-16.

17. Vidal CEL, Bandeira M, Gontijo ED. Reforma psiquiátrica e serviços residenciais terapêuticos. J Bras Psiquiatr. 2008;57(1):70-9.

18. Gentil Filho V. Projeto Delgado, Substitutivo Rocha ou uma lei melhor? Editorial. Rev Bras Psiquiatr. 2001;23(1):1-2.

19. Associação Brasileira de Psiquiatria. Diretrizes para um modelo de assistência integral em saúde mental no Brasil, 2006. Disponível em: http:/www.abpbrasil.org.br/diretrizes_final.pdf. Acessado em: 5/6/2010

20. Brasil, Ministério da Saúde. TabNet Version 2.7. Disponível em: http://www.datasus.gov. br/idb/. Acessado em: 20/1/2010.

21. Andrade LHSG, Lólio CA, Gentil V, Laurenti R. Epidemiologia dos transtornos mentais em uma área definida de captação da cidade de São Paulo, Brasil. Rev Psiquiatr Clin. 1999:26(5):257-61.

22. Almeida-Filho N, Mari JJ, Coutinho E, França JF, Fernandes J, Andreoli SB, et al. Brazilian multicentric study of psychiatric morbidity. Methodological features and prevalence estimates. Br J Psychiatry. 1997;171:524-9.

23. Lima IMN, Alessi NT. O serviço de assistência ambulatorial em saúde mental de uma unidade de saúde do município de Ribeirão Preto. J Bras Psiquiatr. 2002;51(5):269-80.

24. Pitta AMF, Filho JFS, Souza GW, et al. Determinantes da qualidade de serviço de saúde mental em municípios brasileiros - estudo da satisfação com os resultados das atividades desenvolvidas por pacientes, familiares e trabalhadores do serviço. J Bras Psiquiatr. 1995:44(9):441-52.

25. Ribeiro NS. Cinco anos de ambulatório público de saúde mental: 0 ambulatório do Centro Regional de Saúde de Juiz de Fora, MG; 1981/1985. J Bras Psiquiatr. 1994;43(3):163-9. 